\title{
FAULT DETECTION IN SWITCHED RELUCTANCE MOTOR DRIVES USING DISCRETE WAVELET TRANSFORM AND K-MEANS CLUSTERING
}

\author{
${ }^{1}$ Chandrika, V.S. and ${ }^{2}$ A. Ebenezer Jeyakumar \\ ${ }^{1}$ Department of EEE, P.S.V. College of Engineering and Technology, Krishnagiri Dt., Tamilnadu, India \\ ${ }^{2}$ Sri Ramakrishna Engineering College, Coimbatore, Tamilnadu, India
}

Received 2013-11-15; Revised 2013-11-26; Accepted 2013-12-30

\begin{abstract}
This study presents a novel method of detection of inter turn shorts based on $\mathrm{k}$ means clustering technique. In addition to inter turn short detection, the other faults like open, short, phase to phase faults and DC voltage faults are detected through wavelet transforms and $\mathrm{k}$ means clustering. Open and short faults are classified using artificial neural network. All other faults are classified using Support Vector Machines (SVM). Switched reluctance motors are very popular in these days, because of ease in manufacturing and operation. Though an electronic circuit can detect the faults like open and short, the classification cannot be done effectively with electronic circuitry. More over an intelligent method can easily identify the fault and classify and hence the root cause of the fault may be guessed and rectified using this method of classification. This is highly possible with the time localization property of the wavelet transforms. So instant of fault occurrence can be detected along with the type of fault. The information used to include this intelligence in the system are just current waveforms, flux waveforms and torque waveforms. Inter turn shorts are very critical for a long run operation of the motor. Moreover, the early detection minimizes the faulty operation time and ensures the plant stability and saves the life of motor too. Hence an integrated system to detect the major faults under a simulation model has been proposed in this study.
\end{abstract}

Keywords: Discrete Wavelet Transforms (DWT), Inter Turn Shorts, K-Means Clustering, Switched Reluctance Motor (SRM), Support Vector Machines (SVM)

\section{INTRODUCTION}

The applications of SRM in aircraft and industrial automations applications are enormous and need a perfect flaw free operation to obtain the required electrical and mechanical outputs from the motor (Gameiro and Cardoso, 2012). The absence of rotor windings and permanent magnets in rotor makes the manufacturing of SRM easy and hence the SRM is very popular in market based on commercial aspects too. The special feature of SRM is that, a particular phase of SRM is not influenced by the other phase and is very negligible. Hence, the motor continues to rotate even at faulty conditions but it might not produce the exact required output parameters based on mechanical aspects.
So, early detection of the faults in SRM is mandatory. The salient pole configuration of the SRM is responsible for ripples in torque, anyhow that can be minimized using the works in (Xue et al., 2009). The major issue with faulty operation is that, though the motor continues to rotate, the mechanical forces become imbalanced and the mechanical power decrease proportional to the number of phases disconnected from the circuit. Open circuit faults have not been given much importance in earlier literatures except (Gameiro and Cardoso, 2012). Open circuit can be easily identified with the presence or absence of the phase current. A typical electronic circuit would do it. But the circuit fails to classify the faults if the numbers of faults are more and more over the time instant of fault occurrence is never known with circuit P.S.V. College of Engineering and Technology, Krishnagiri Dt., Tamilnadu, India 
based detection. In addition to the above said draw backs of circuit based detection, the circuits needs the sensors which are likely to fail. Also the number sensors to be used are proportional to the number of phases, which considerably increases the cost of the system. Hence a processor based intelligent device may be suitable at these circumstances. Fault tolerant systems are abundant in market, in which the motor can continue with its operation even at faulty conditions like, open, short and phase to phase shorts as given in (Sivakumar and Parvathi, 2013). This study would be a main source for all other further AI based fault detection systems. But such models were unable to classify the various faults, so remedial action could not be taken against the faults. The authors of this study feel that apart from the fault detection, fault classification becomes essential in order to impart intelligence to the machines. As in (Paramasivam and Arumugam, 2004) certain works had been done using fuzzy controller to stablize the SRM. The papers (Gameiro and Cardoso, 2010; 2012; Schinnerl and Gerling, 2009; Terec et al., 2011; Miremadi et al., 2013), discusses about the various power converters and faults likely to occur and methods to detect the faults. To the best of the author's knowledge, clustering algorithms and SVM based classification techniques had not been used in SRM fault detection. This study has been organized as follows: Section 2 to 5 , describes the concept behind the wavelet transforms, BPN classification techniques, $\mathrm{K}$ means clustering and SVM classification techniques respectively. Section 6 discusses about the proposed method and the simulation outputs are discussed in section 7.

\section{DISCRETE WAVELET TRANSFORM}

The basic concept of wavelet transforms is to simultaneously pass a signal $\mathrm{s}(\mathrm{t})$ into a low pass and high pass filter. The lower band and higher band are called as called as Approximation coefficients and Detail coefficients respectively. Defect identification in composite materials has been discussed in (Aicha et al., 2011) and this will be inspiation for any further wavelet based defects and fault analysis using wavelets. The wavelet transform is nothing but transforming a time domain signal into a set of orthogonal or ortho normal functions, namely the wavelets. The functions can be continuous (continuous wavelet transform) or discrete (discrete wavelet transform). The major aim of this work is to monitor the performance of SRM and its integrity. so, it is necessary to assess the machine condition. The main idea is to utilize the steady-state frequency components of the stator electrical quantities. These frequency components of stator waveforms can include current, flux, torque are used to detect the various faults. Unlike fourier transorms, wavelet transforms results in the representation of original signal with respect to time and frequency as well. This property is called as time and frequency localization. So the time instant of the fault occurrence is highly possible when wavelet transforms are used. Wavelet transforms may a good choice for stationary signal processing. With time localization property it is possible to find the instant of fault occurring instant. While decomposing any time signal into its wavelet transforms, any wavelet family may be used and any shape which satisfies, two properties viz., (1) Mean value of the wavelet is zero (2) Energy of the singal is finite. As power system disturbances are subject to transient and non-periodic components, the DFT is not adequate for signal analysis (Akansu et al., 2010).

\section{BACK PROPOGATION NETWORK}

It is a supervised learning method and is an implementation of the Delta rule. Back Propagation is a type of neural network method applicable for multi layered ANN. It consists of basic processes like training and testing. While training process, the errors between the target value and the input value are propagated back to the input. Back propagation method follows a scheme of changing the weights in a feed forward network, with differentiable activation function units, to learn a training set of input-output examples. BPN reduces the total squared error of the output computed by the net. This called as supervised learning method. As shown in Fig. 1, the general architecture of BPN, the input layer, hidden layer and the output layers are connected together sequentially by means of interconnection weight. It is always a good practice to maintain the number of neurons in hidden layer is more than the number of neurons in the input layer. But this results usually in the higher computational complexity of the network. So it is transparent that, the time of training and hence the time for minimization of error becomes high. For small data sets this may not be problem. In our work, a network is constructed with three input neurons, three hidden layer neurons and three output neurons. Three input values to the input layer represents the values of detail coefficients obtained through a 11 level decomposition. The three neurons at the output layer represents three findings like normal, open and short conditions. Instead of applying the whole length of the test voltages or current waveforms, it is enough to apply only the magnitude of the detail coefficients as training and testing parameter. 


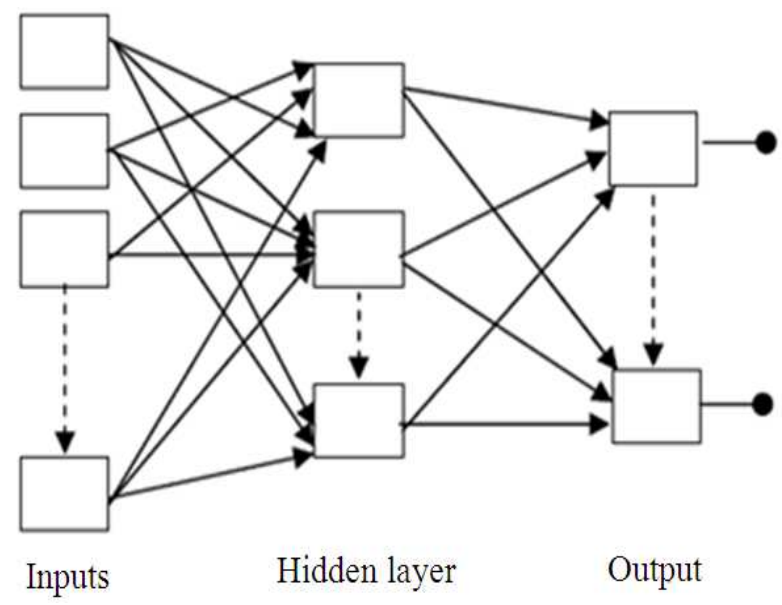

Fig. 1. Architecture of BPN

Any how, in our work, the features are extracted from wavelet transforms and $\mathrm{k}$ means clustering algorithm, hence training and testing time is very less and negligible. The training algorithm of back propagation involves four stages, viz:

- Assigning random weights to all paths

- Calculating the output magnitudes

- Sending back the errors

- Reconstructing the weight values

The trainig process is performed for a predermined number of number of epochs. The training stopping criteria is based on either the number of iterations reaches first or the minimum goal error is reaches first. Once the training is over, the system stores the weight values in memory and used when the test input is applied online during the functioning of the SRM. Since the training is done offline, the fault detection speed is high.

\section{K-MEANS CLUSTERING}

Clustering is a method of grouping similar data into various groups based on the amplitude of the data points. This is an iterative scheme to find the local minimal solution. This clustering method has been clearly shown in (Shanmugam et al., 2011). Optimal placement of the center at centroid is the technique behind this algorithm. Let us suppose that $\mathrm{N}$ numbers of data points are the outcome of an experiment. These data points are clustered into $\mathrm{K}$ number of clusters, with each cluster consisting the number of elements which depends on the value of the data points. Mathematical investigation of $\mathrm{k}$ means algorithms is beyond the scope of this study. In our work, the feature vectors for detecting the DC voltage fault and inter turn faults are extracted from using this $\mathrm{k}$ means clustering. The corresponding waveforms and results are shown in section 7 .

\section{SUPPORT VECTOR MACHINE BASED CLASSIFICATION}

The basic idea behind the SVM classification technique is to identify the class of the input test vectors. This is a supervised learning algorithm, where the training vectors are used to train the system to map these training vectors in a space with clear gaps between them using some standard kernel functions and the input test vectors are mapped on to the same space to predict the possible class. The choice of the kernel functions have been discussed in (Gomathi and Thangaraj, 2010). Given some training data D, a set of $\mathrm{n}$ points of the form Equation (1):

$\mathrm{D}=\left\{\left(\mathrm{x}_{\mathrm{i}}, \mathrm{y}_{\mathrm{i}}\right) \mid \mathrm{x}_{\mathrm{i}} \in \mathbb{R}^{\mathrm{p}}, \mathrm{y}_{\mathrm{i}} \in\{-1,1\}\right\}_{\mathrm{i}}^{\mathrm{n}}=1$

where, $\mathrm{y}_{\mathrm{i}}$ is either belonging to the class 1 or class-1, which means the class which the point $\mathrm{X}_{\mathrm{i}}$ belongs. Each $\mathrm{X}_{\mathrm{i}}$ is a p-dimensional real vector. While classifying, it is essential to find the maximum possible margin hyper plane that segments the data sets having $y_{i}=1$ from those data sets having $y_{i}=-1$. So any hyper plane can be written as data sets $X$ satisfying maximum possible margin hyperplane and margins for an SVM trained with samples from two classes. Samples exactly on the margins are called the support vectors Equation (2):

$$
\text { W.X }-\mathrm{b}=0
$$

where, . denotes the dot product and $\mathrm{W}$ is the normal vector to the hyper plane. The term $b /\|w\|$ determines the offset of the hyper plane from the origin along the normal vector $\mathrm{W}$. In case, the training data sets are linearly separable, then two hyper planes can be constructed in such a way that they group the data sets and there are no points between them and then tried to increase the distance among them. The region surrounded by them is called "margin". These hyper planes can be described by the Equation ( 3 and 4 ):

$\mathrm{w} \cdot \mathrm{x}-\mathrm{b}=1$

And:

$\mathrm{w} \cdot \mathrm{x}-\mathrm{b}=-1$ 


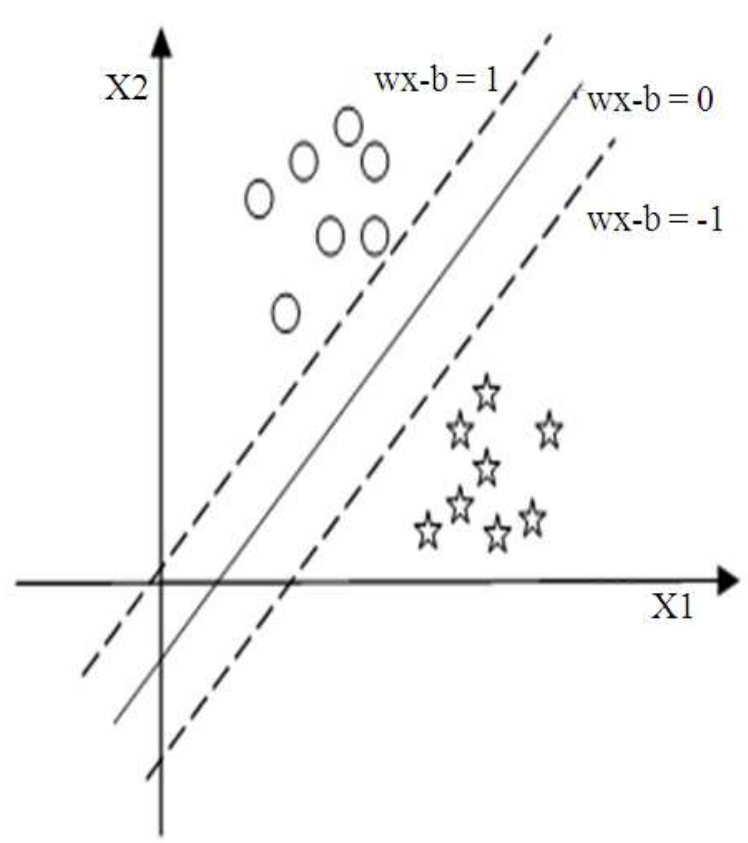

Fig. 2. SVM Scenario

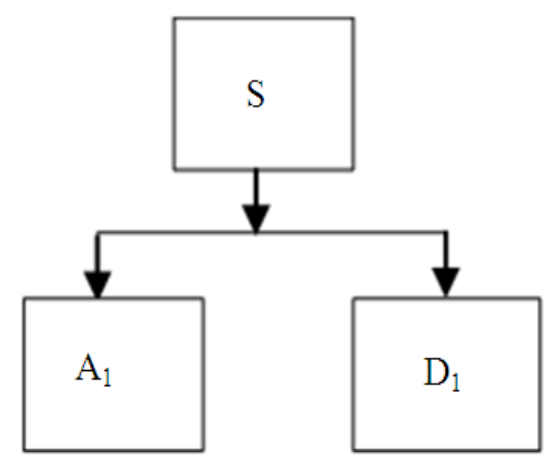

Fig. 3. Wavelet transform schematic

Figure 2 shows the process of how two data sets (one represented by circles and the other set by stars) are classified in two different regions while a linear kernel is used for classification. In the testing phase, the data points $x_{i}$ are separated using the following constraints w. $x i-b \geq 1$ for $x_{i}$ of the first class or $w . x i-b \leq 1$ for $x_{i}$ of the second class.

\section{PROPOSED FAULT DETECTION METHOD}

There exist two types of motoring operation based on the health of motors, Normal operation and faulty operation. These modes of operation are well discussed in (Gameiro and Cardoso, 2012). The major contribution in this study is to detect the various faults like open, short, phase to phase short, dc voltage fault and interturn shorts. This integrated method of detecting the fault is achieved through an efficient method of feature selection and classification. The basic idea behind the detection of open and short fault is to detect the amplitude of the detail co efficient. Wavelet transforms are sensitive to frequency changes in periodic waves. Though open and short circuits can be detected easily with simple electronic circuitry, any such basic systems fail to classify the faults and fail to record the time instant of such open and short fault occurrences. Also, the basic electronic circuitry fails to detect automatically, the phase in which the fault occurred. Knowing the time instant of fault occurrences is very essential in air craft systems and high end research applications. Hence wavelet transforms would be the best option. DC voltage shift directly reflects in the magnitude of the torque ripples hence the range of torque ripples can be used to detect the range of DC voltage. These online values of torque data is clustered and features are classified to find the voltage fault. This method is very useful when the electrical drive circuit is far away from the worksite of the motor. The phase to phase short faults usually create a flux to deviate from its healthy condition. These flux values are clustered to find the mean value of the data points and number of data points in each class. This is done using $\mathrm{k}$ means clustering. For the detection of inter turn short the same method is followed to arrive the clustered values and their counts. These clustered values are classified using Support Vector Machines (SVM). An SRM of $8 / 6$ is run at steady state first and then, the decomposition of the signal into 11 levels and hence 11 frequency bands (Fig. 3) are obtained by means of successive high pass and low pass filtering of the time domain signal. For simplicity, only one decomposition is shown in the figure.

The original time domain signal $\mathrm{S}$ is first filtered with a high pass filter where detail efficient $D_{1}$ is obtained and simultaneously through a low pass filter where approximation coefficient $A_{1}$ is obtained. It can be repeated for further decomposition using $A_{1}$. At each level, a down sampling is done. A simulation model as shown in the Fig. 4 is constructed and simulated with various faults like open, short, phase to phase, DC voltage and inter turn faults. The saved values of the line 
currents were exported in MATLAB for further stage to classify to know which phase of the SRM is with short circuit or open circuit fault. 11 level one-dimensional discrete wavelet analysis was performed using the wavedec function. The daube-chies wavelet, db1 type wavelet was selected. As it will be seen the difference signal at the 11 th level of decomposition $\left(\mathrm{d}_{11}\right)$ can be used for fault detection of the short circuited or open circuited faulty drive. The same is re-constructed, using the wrcoef function of the MATLAB toolbox. Next in Fig. 6 and 8, the obtained $d_{11}$ wave-let coefficient's variation versus time are given for the healthy and faulty SRM when loaded torque is 0.9. The magnitude of the $\mathrm{d}_{11}$ coefficient is always higher at faulty conditions. At no-load the effect of the fault is not as serious as in the case of the rated load, since the currents in the stator windings are small. Therefore the most relevant results were obtained at great loads, especially near the rated load. After the steady state is reached a faulty condition is simulated by short circuiting or open circuiting any switch, then corresponding current waveforms are transformed into wavelet domain. With eleventh Detail coefficient, it is possible to detect the short and also the time instant of short. This time localization property is helpful to analyse the root cause for the fault. As it can be seen, the waveforms in the output figures show that, only at the faulty conditions, the value of $\mathrm{d}_{11}$ coefficient exceeds a threshold being 40 in our case.

\section{SIMULATION AND OUTPUTS}

Figure 4 shows the simulation model of the proposed fault detection method with all five faults. First the SRM drive is simulated at healthy condition and the corresponding Flux, Stator current, Torque variations and Speed outputs are obtained. Then the open, short, phase to phase, DC voltage and inter turn faults are created and the same parameters, Flux, Stator current, Torque variations and Speed are shown.

\subsection{Healthy Conditions}

A Matlab simulink model of the SRM drive with $8 / 6$ configuration has been designed and simulated with all the switches at perfect healthy conditions. The parameters have been shown at steady state. Load torque is set as 0.9. Fig. 5 shows the output of the healthy SRM drive with a speed of $2000 \mathrm{rpm}$. The parameters like, Flux, Stator current, Torque variations and Speed are shown. Corresponding $d_{11}$ coefficient has been shown in Fig. 6.

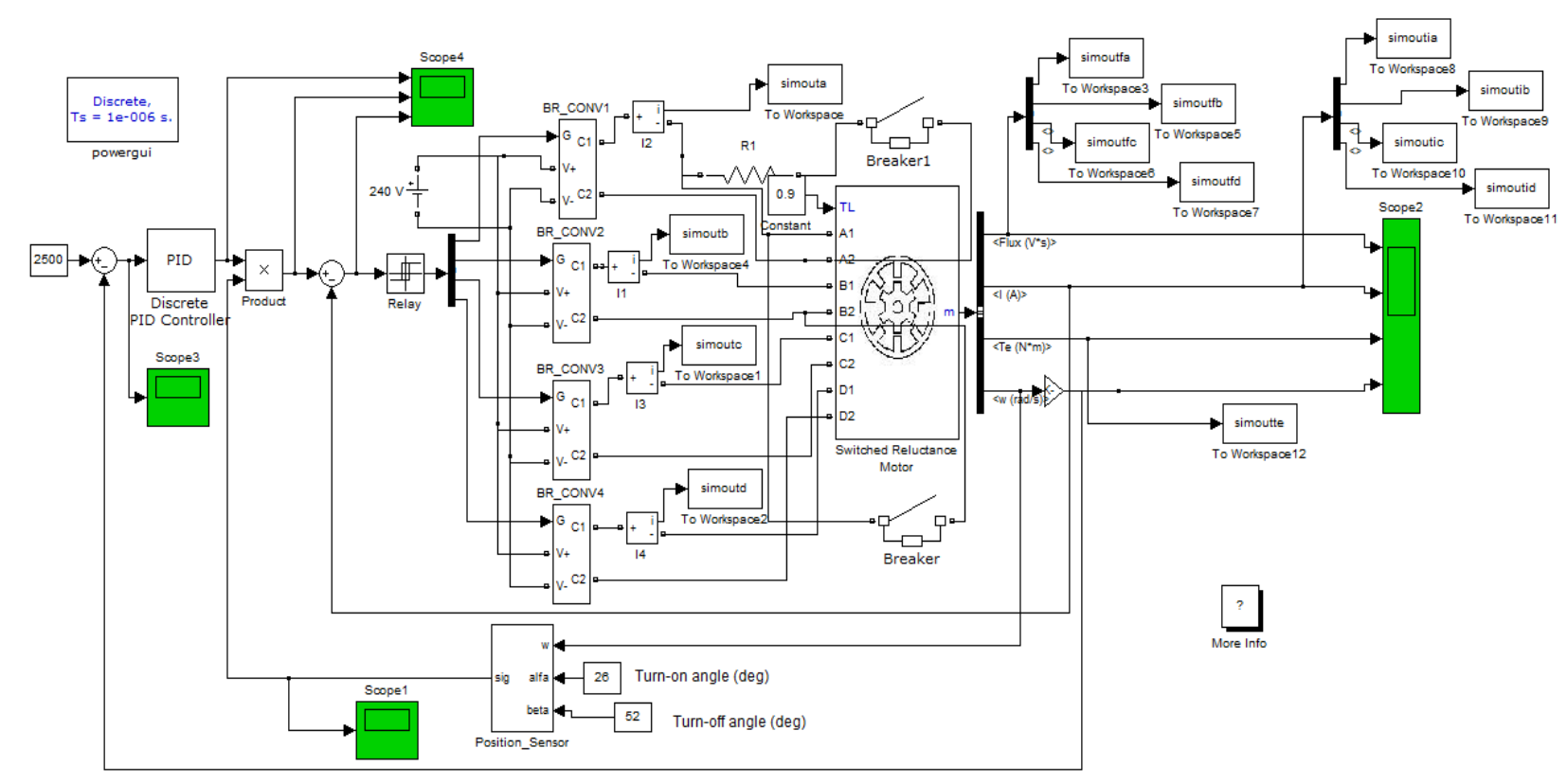

Fault detection in Current-controlled 8/6 Switched Reluctance Motor drive

Fig. 4. Simulation model of SRM 


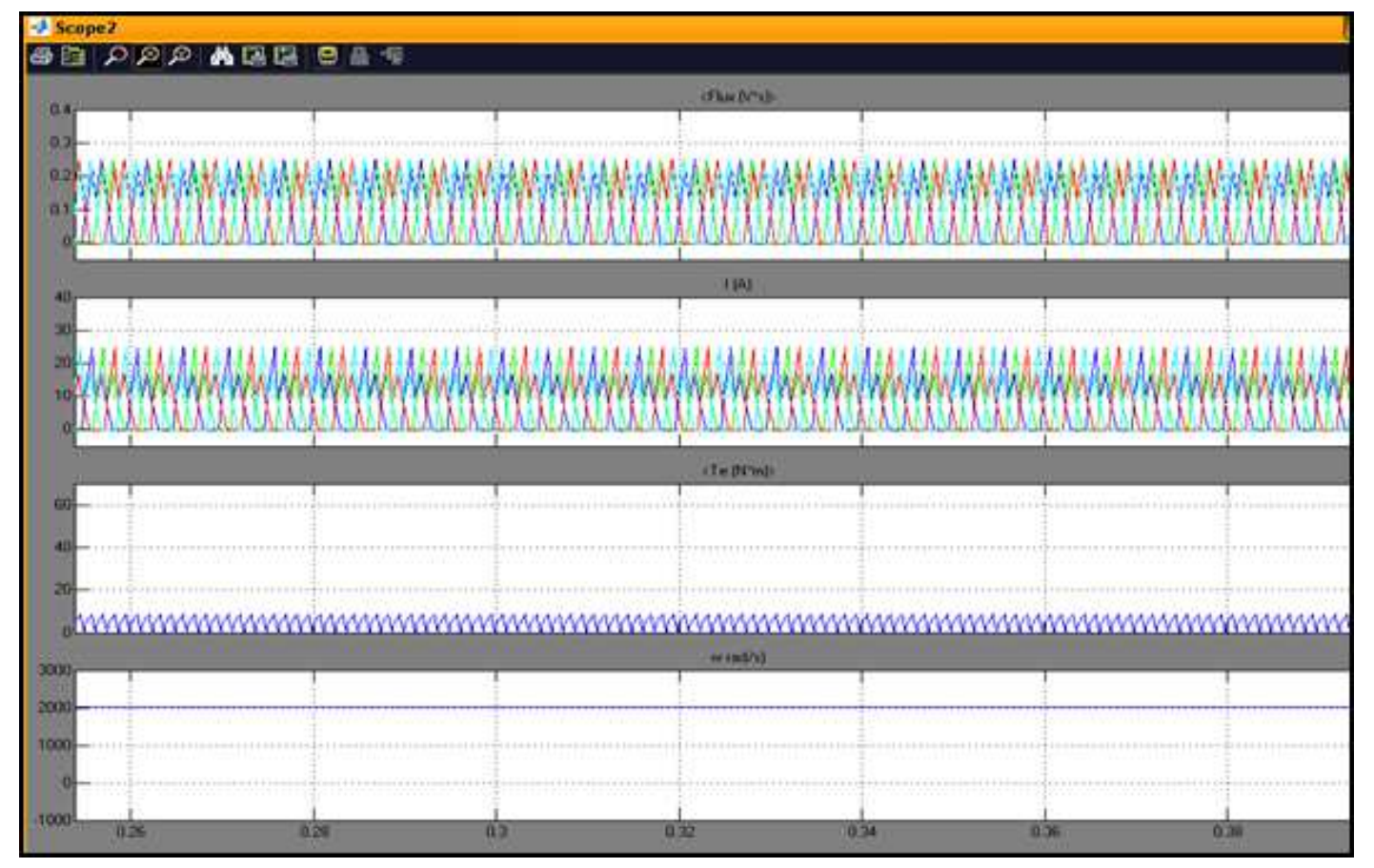

Fig. 5. Steady state waveforms of flux, current, torque and speed at healthy conditions
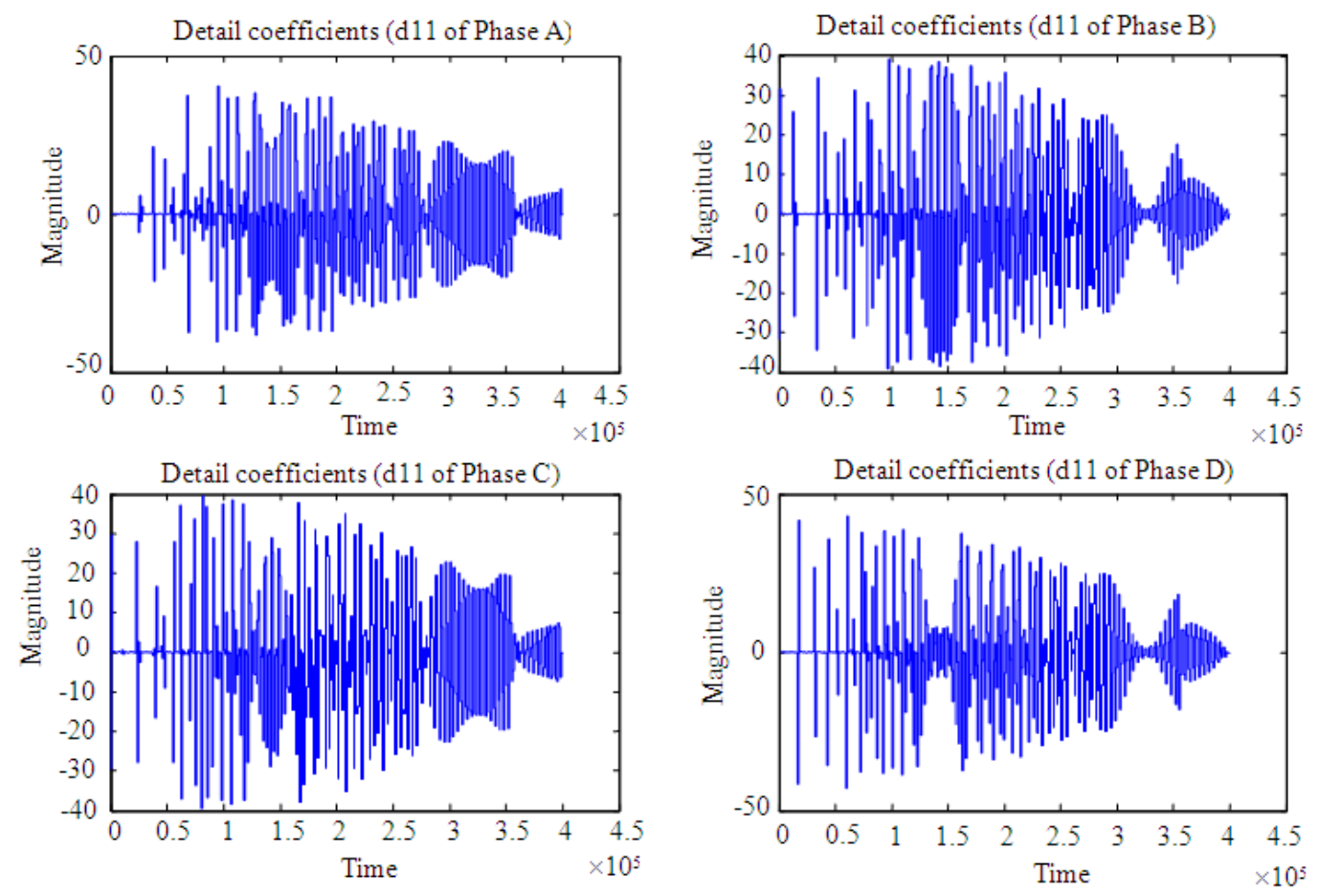

Fig. 6. Detail co efficient of Phase A, Phase B, Phase C and Phase D at healthy condition 


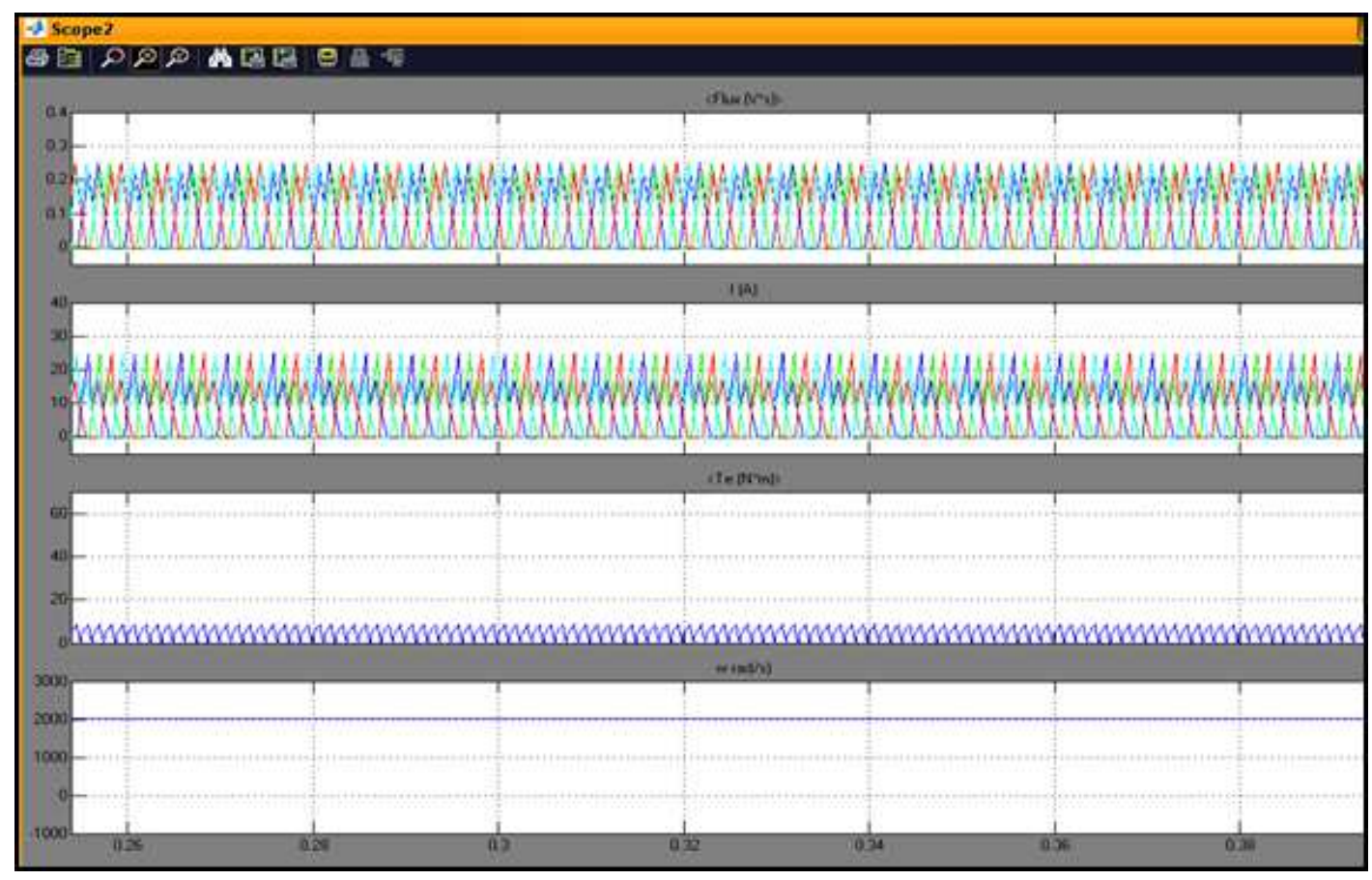

Fig. 7. Steady state waveforms of flux, current, torque and speed with phase a short and phase c open
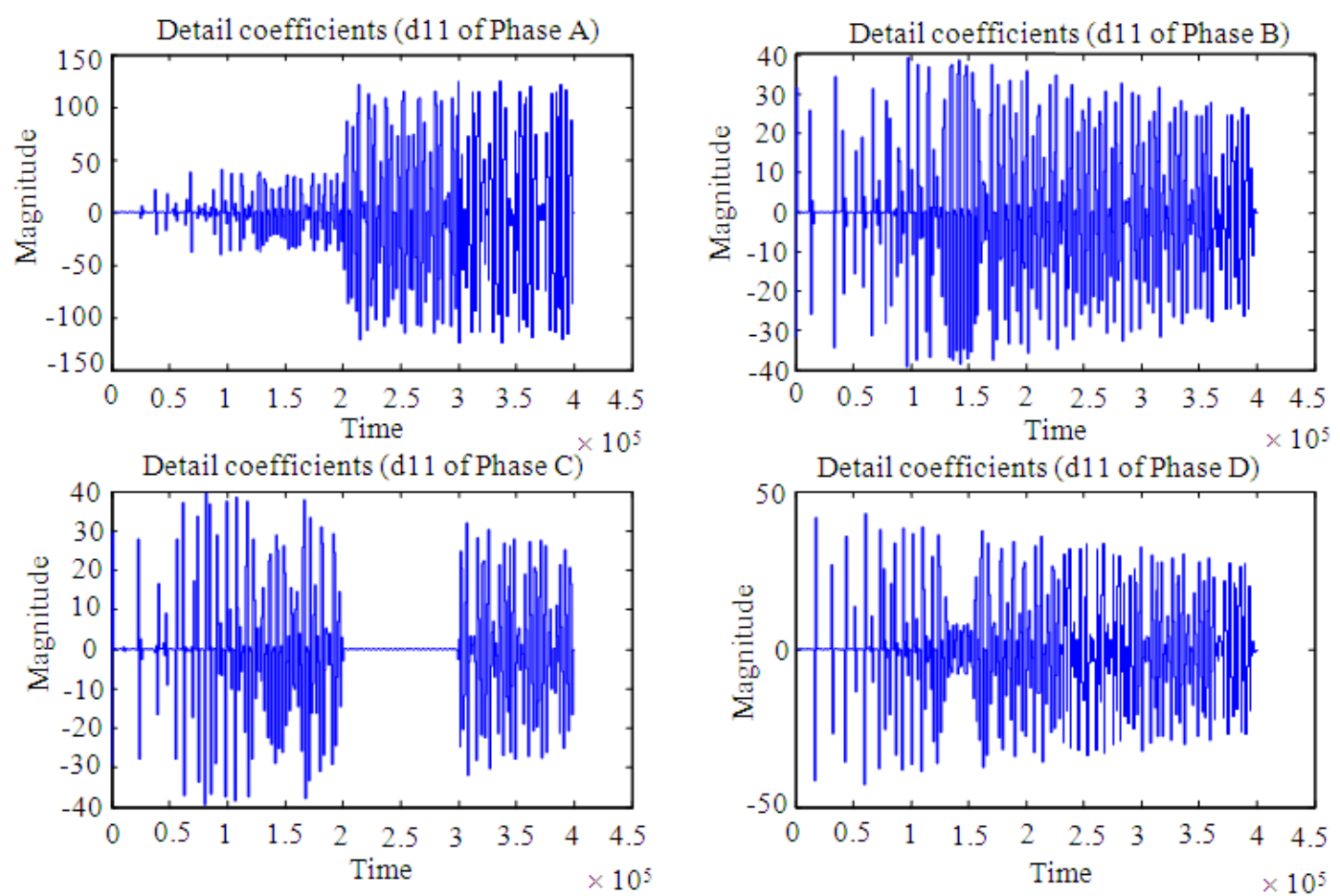

Fig. 8. Detail co efficient of phase a(short), phase b(normal), phase c(open) and phase d(normal) 


\subsection{Open and Short Conditions}

Phase A and Phase $\mathrm{C}$ are short circuited and open circuited respectively at $t=0.2 \mathrm{~s}$. Figure 7 shows the output of the SRM drive with a speed of $2000 \mathrm{rpm}$ under short circuit and open circuit conditions. It is seen from the figure that, the torque and the speed becomes oscillatory. More over the flux of short circuited coils saturates to the maximum possible value. Corresponding $d_{11}$ coefficient has been shown in Fig. 8 .

It is observed from Fig. 8, that the detail co efficient value exceeds a threshold 40 and reaches 100 for Phase A. Also the time instant of the fault is found out as 0.2041 for phase A and 0.2058 for phase C. Further the maximum values of details coefficient is given by BPN classification technique to find the nature of fault in specific coils of the corresponding phases.

\subsection{Inter Turn Short Conditions}

Inter turn shorts may occur in an SRM, due to environmental conditions or due to aging of the motors. Though motor continues to run with inter turn shorts, the problem could become serious because of ripples in torque. Inter turn shorts were created between the times $0.15 \mathrm{~s}$ to $0.2 \mathrm{~s}$, with five different classes like $0-10,30$ $40,50,80-90$ and $90-100 \%$. The flux waveforms were observed and clustered using $\mathrm{k}$ means algorithm with number of clusters $\mathrm{k}=5$.

It is observed that, when the inter turn short percentage increases, the flux wave try to saturate, anyhow this saturation amplitude differs based on the amount of shorted turns. This amplitude change in particular interval is exported to $\mathrm{k}$ means algorithm and clustered into 5 groups. The number of elements in each group purely depends on the amount of the flux saturation. This is well classified using a multi class Support Vector Machines. In the proposed work, inter turn shorts were considered in phase A alone. The faulty waveforms are shown in the Fig. 9.

\subsection{Phase to Phase Short Conditions}

Phase to phase shorts were created externally, (in our case, Phase A is shorted with Phase B) during the time intervals $0.1 \mathrm{~s}$ to $0.15 \mathrm{~s}$. Unlike inter turn shorts, here, the faults need not be classified, it would be enough to identity the fault. The SVM network, already trained with flux waveforms of healthy motor is tested with the obtained clusters and the number of elements in each cluster obtained from the flux waveforms at online. Here number of clusters are taken as $\mathrm{k}=10$. The faulty waveforms are shown in the Fig. 9.

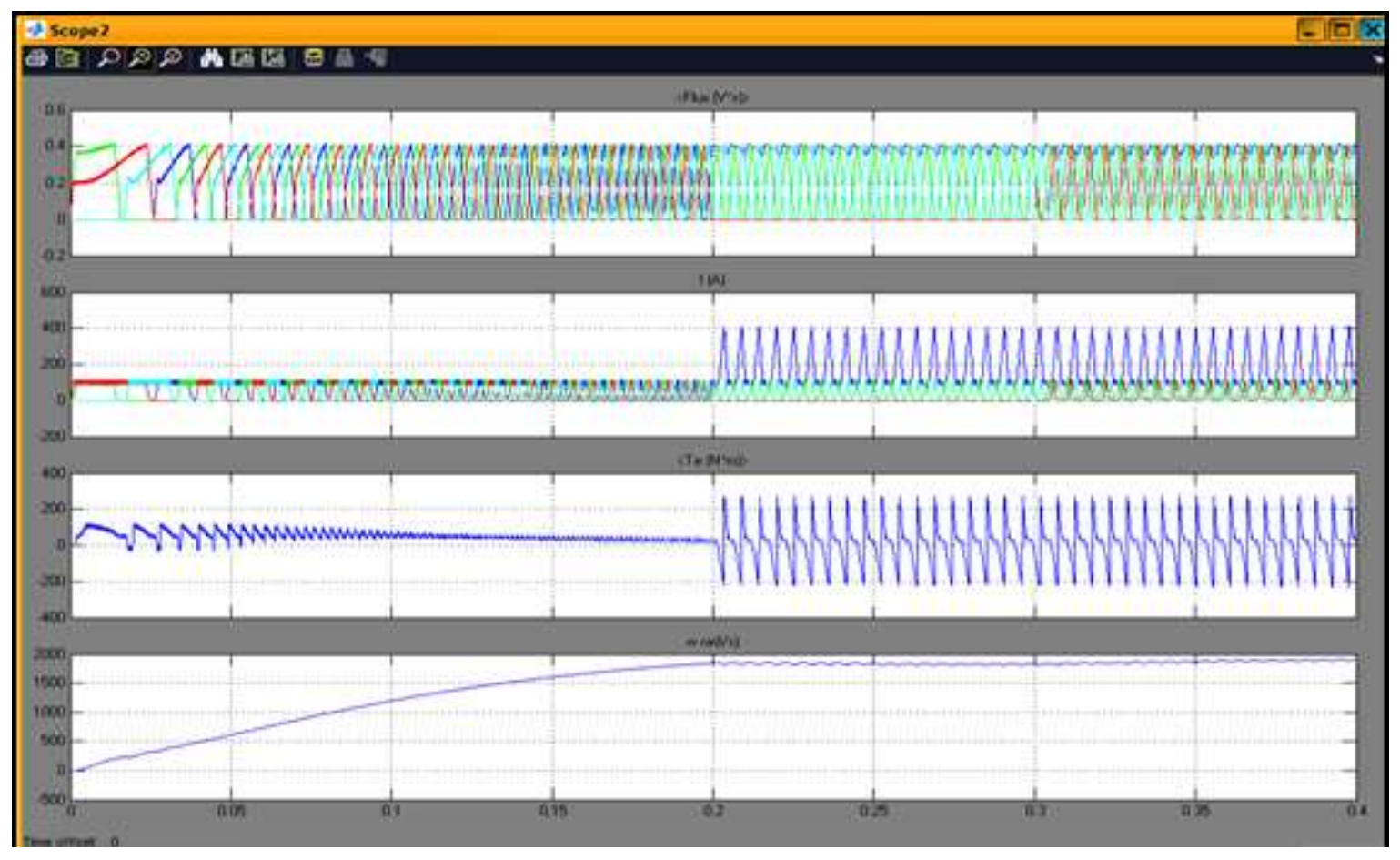

Fig. 9. Waveforms of flux, current, torque and speed at inter turn, phase to phase short and dc voltage fault 


\subsection{Voltage Fault Conditions}

As in the case of phase to phase shorts, in this DC voltage faults, $\mathrm{k}=10$ is chosen to cluster the torque wave-forms. Two voltage conditions of $240 \mathrm{~V}$, a full rated voltage and $120 \mathrm{~V}$, half of the rated voltages were applied to excite the SRM. Based on the number of elements in cluster, Classification using SVM is performed to identify the DC bus fault. The faulty waveforms are shown in the Fig. 9.

\section{CONCLUSION}

In this study, a DWT based method to detect the short circuit and open circuit fault and classification based on Back propagation network has been imple-mented. Also, time of fault occurrence has also been found out in this work. Hence, earlier detection of short circuit increases the life time of the SRM. In addition to this, DC Voltage fault, Phase to phase short and inter turn shorts are detected using $\mathrm{k}$ means clustering along with the percentage of inter turn shorts using SVM. Implementation of this simulation as a real time system requires a high speed processor to perform all mathematical calculations along with high speed Analog to digital converters at online. In our proposed method, when short circuit occurs, our simulation result may show both short circuit and interturn short of $100 \%$, this could be serious limitation in our work. Any how, the future extension of this work may be concentrated on this issue.

\section{ACKNOWLEDGEMENT}

Our thanks to the support extended by the P.S.V College of Engineering and Technology, Krishnagiri.

\section{REFERENCES}

Aicha, Y., S.C. Med and O.A. Chellil, 2011. Identification of defects in composite materials using an improved wavelet analysis algorithm. Phys. Int., 2: 50-56. DOI: 10.3844/pisp.2011.50.56

Akansu, A.N., W.A. Serdijn and I.W. Selesnick, 2010. Emerging applications of wavelets: A review. Physical Commun., 3: 1-18. DOI: 10.1016/j.phycom.2009.07.001

Gameiro, N.S. and A.J.M. Cardoso, 2012. A New method for power converter fault diagnosis in SRM drives. IEEE Trans. Indus. Applic., 48: 653-662. DOI: 10.1109/TIA.2011.2180876
Gameiro, N.S. and M.A.J. Cardoso, 2010. Power converter fault diagnosis in SRM drives based on the DC bus current analysis. Proceedings of the 19th International Conference on Electrical Machines, Sept. 6-8, IEEE Xplore Press, Rome, pp: 1-6. DOI: 10.1109/ICELMACH.2010.5608258

Gomathi, M. and P. Thangaraj, 2010. A computer aided diagnosis system for lung cancer detection using support vector machine. Am. J. Applied Sci., 7: 1532-1538. DOI: 10.3844/ajassp.2010.1532.1538

Miremadi, A., H. Torkaman and A. Siadatan, 2013. Maximum current point tracking for stator winding short circuits diagnosis in switched reluctance motor. Proceedings of the 4th Power Electronics, Drive Systems and Technologies Conference (PEDSTC), Feb. 13-14, IEEE Xplore Press, Tehran, pp: 83-87. DOI: 10.1109/PEDSTC.2013.6506678

Paramasivam, S. and R. Arumugam, 2004. Real time hybrid controller implementation for switched reluctance motor drive. Am. J. Applied Sci., 1: 284294. DOI: 10.3844/ajassp.2004.284.294

Schinnerl, B. and D. Gerling, 2009. Analysis of winding failure of switched reluctance motors. Proceedings of the IEEE International Electric Machines and Drives Conference, May 3-6, IEEE Xplore Press, Miami, FL., pp: 738-743. DOI: 10.1109/IEMDC.2009.5075287

Shanmugam, N., A.B. Suryanarayana, S. TSB, D. Chandrashekar and C.N. Manjunath, 2011. A novel approach to medical image segmentation. J. Comput. Sci., 7: 657-663. DOI: 10.3844/jcssp.2011.657.663

Sivakumar, M. and R.M.S. Parvathi, 2013. Particle swarm and neural network approach for fault clearing of multilevel inverters. Am. J. Applied Sci., 10: 579-595. DOI: 10.3844/ajassp.2013.579.595

Terec, R., I. Bentia, M. Ruba, L. Szabo and P. Rafajdus, 2011. Effects of winding faults on the switched reluctance machine's working performances. Proceedings of the 3rd IEEE International Symposium on Logistics and Industrial Informatics (LINDI), Aug. 25-27, IEEE Xplore Press, Budapest, pp: 143-148. DOI: 10.1109/LINDI.2011.6031137

Xue, X.D., K.W.E. Cheng and S.L. Ho, 2009. Optimization and evaluation of torque-sharing functions for torque ripple minimization in switched reluctance motor drives. IEEE Trans. Power Electron., 24: 2076-2090. DOI: 10.1109/TPEL.2009.2019581 